Dynamical Jahn - Teller effect, double exchange and the isotope shift in the manganites: a toy model

This article has been downloaded from IOPscience. Please scroll down to see the full text article.

1998 J. Phys.: Condens. Matter 10 L501

(http://iopscience.iop.org/0953-8984/10/29/003)

View the table of contents for this issue, or go to the journal homepage for more

Download details:

IP Address: 128.206.162.204

The article was downloaded on 22/12/2010 at 20:56

Please note that terms and conditions apply. 
LETTER TO THE EDITOR

\title{
Dynamical Jahn-Teller effect, double exchange and the isotope shift in the manganites: a toy model
}

\author{
S Satpathy \\ Department of Physics and Astronomy, University of Missouri, Columbia, MO 65211, USA
}

Received 12 June 1998

\begin{abstract}
A minimal model, consisting of a $4 \times 4$ Hamiltonian and pertaining to a single $\mathrm{Mn}-\mathrm{O}-\mathrm{Mn}$ bond in the manganites, is introduced and solved to elucidate the physics of the dynamical Jahn-Teller effect on the double-exchange (DEX) interaction. The effect originates from the coupling of the motion of the bridging oxygen atom to the Mn electrons. It is shown that (i) the DEX interaction is considerably reduced by the dynamical Jahn-Teller effect and (ii) this reduction depends on the oxygen mass leading to an isotope shift in the Curie temperature, $T_{c} \propto M^{\alpha}$, with $\alpha \sim 0.2$. The isotope shift is found to depend sharply on the electron hopping parameter $t$. The exact results are also compared to the results obtained from both the adiabatic and the diabatic approximations.
\end{abstract}

The conventional theory of magnetism ignores the effect of lattice motion on magnetic interaction. While this is true for many systems, for others, coupling to the lattice degrees of freedom can have an important effect on the magnetic interaction. The recent observation of the isotope shift of $T_{c}$ in the lanthanum manganites is an example of this [1]. Another interesting experiment in this context is the work of Zhou and Goodenough [2] indicating that the dynamical Jahn-Teller (JT) coupling affects the transport properties in the manganites in a regime where the static JT distortions are even suppressed. A summary of the electron-ion coupling effects in the manganites is given by Millis [3]. Here we introduce and solve a simple minimal model of electron-ion coupling on a single $\mathrm{Mn}-\mathrm{O}-\mathrm{Mn}$ bond to illustrate the effect of the dynamical JT coupling on the DEX interaction $[4,5,6]$. Being simple and exactly solvable, the model allows us to compare the accuracy of the various approximation methods and at the same time provides us with a pedagogical explanation of the observed isotope shift.

The model consists of a single itinerant electron moving between two Mn atoms resulting in a fluctuating valence of either $\mathrm{Mn}^{+3}$ or $\mathrm{Mn}^{+4}$. Being a JT ion, $\mathrm{Mn}^{+3}$ produces a static distortion of the surrounding $\mathrm{MnO}_{6}$ octahedron as sketched in figure 1. As the electron moves from one $\mathrm{Mn}$ to the other, the $\mathrm{O}_{6}$ distortion has a tendency to move along with it, so that the motions of the electron and the oxygen octahedra become intricately coupled, leading to a modification of the magnetic interaction and the observed isotope effect.

The minimal model that describes this physics consists of two electron orbitals on the $\mathrm{Mn}$ atoms, denoted by $|a\rangle$ and $|b\rangle$, and two nuclear basis states $|\alpha\rangle$ and $|\beta\rangle$, corresponding to the oscillations of the oxygen octahedra about the two stable configurations $\alpha$ and $\beta$ as shown in figure 1. The Schrödinger equation for the coupled system is given by $H|\psi\rangle=E O|\psi\rangle$ where the Hamiltonian is

$$
H=H_{e}+H_{n}+H_{e-n}
$$






Figure 1. Model for double exchange in the presence of Jahn-Teller coupling. The configuration shown is $|\alpha a\rangle$, which corresponds to electron in state $|a\rangle$ and the oxygen octahedra rattling around the equilibrium configuration shown by the full lines. This rattling is described by the nuclear wavefunction $|\alpha\rangle$ taken as the ground-state wavefunction of the corresponding simple harmonic oscillator. The state $|\alpha a\rangle$ is not an eigenstate of the system so that coupling to other configurations, e.g., $|\alpha b\rangle$, can occur, leading to a coupling between the nuclear and electronic degrees of freedom.

and $O$ is the overlap matrix. In our model, the Hamiltonian matrix elements are taken as

$$
\begin{array}{ll}
\left\langle a\left|H_{e}\right| a\right\rangle=\left\langle b\left|H_{e}\right| b\right\rangle=\epsilon \quad & \left\langle a\left|H_{e}\right| b\right\rangle=-t \\
\left\langle\alpha\left|H_{n}\right| \alpha\right\rangle=\left\langle\beta\left|H_{n}\right| \beta\right\rangle=\epsilon^{\prime} & \left\langle\alpha\left|H_{n}\right| \beta\right\rangle=-T \\
\left\langle\alpha a\left|H_{e-n}\right| \alpha a\right\rangle=\left\langle\beta b\left|H_{e-n}\right| \beta b\right\rangle=\epsilon^{\prime \prime} & \\
\left\langle\alpha b\left|H_{e-n}\right| \alpha b\right\rangle=\left\langle\beta a\left|H_{e-n}\right| \beta a\right\rangle=\Delta &
\end{array}
$$

while the overlap matrix elements are

$$
\langle a|O| b\rangle=\delta_{a b} \quad\langle\alpha|O| \alpha\rangle=\langle\beta|O| \beta\rangle=1 \quad\langle\alpha|O| \beta\rangle=F .
$$

The electronic part is of tight-binding form with a hopping integral between the two $\mathrm{Mn}$ orbitals, which in the context of the solid comes from the overlap of the Wannier functions. Of the above parameters, we take $\epsilon=\epsilon^{\prime}=\epsilon^{\prime \prime}=0$, which redefines the zero of the energy. We also take $T=0$ meaning that the oxygen atoms cannot switch between configurations $\alpha$ and $\beta$ without involving electronic motion. The off-diagonal matrix elements of $H_{e-n}$, such as $\left\langle\alpha a\left|H_{e-n}\right| \beta a\right\rangle$ are in general small and are omitted for simplicity.

The Hamiltonian parameters are: (i) the electronic hopping integral $t \approx 0.15 \mathrm{eV}$, (ii) the quantity $\Delta$ which is the energy of the 'wrong' configuration of the oxygen octahedra with respect to the electron position, and (iii) the stiffness constant $K$ of the simple harmonic oscillator (SHO) potential well for oxygen vibrations. From band calculations [7] and crystal structure data [8], we estimate $K \equiv m \omega^{2} \approx 20 \mathrm{eV} \AA^{-2}$, where $m$ is the oxygen mass. This is consistent with a static Jahn-Teller displacement of the oxygen atoms by about $0.1 \AA$ in $\mathrm{LaMnO}_{3}$. We take $\Delta=4 \Delta_{J T}$, where $\Delta_{J T} \approx 0.15 \mathrm{eV}$ for the manganites. The overlap $F$ between the two nuclear wavefunctions is the 'Frank-Condon factor'. If for states $|\alpha\rangle$ and $|\beta\rangle$ one takes the SHO ground-state wavefunctions, the overlap is given by $F=\exp \left(-\Delta_{J T} / \frac{1}{2} \hbar \omega\right)$.

With these, the Hamiltonian and overlap matrices in the combined electronic-nuclear 
basis set, $|\alpha a\rangle,|\alpha b\rangle,|\beta a\rangle$, and $|\beta b\rangle$, are given by

$$
H=\left(\begin{array}{cccc}
0 & -t & 0 & -F t \\
-t & \Delta & -F t & 0 \\
0 & -F t & \Delta & -t \\
-F t & 0 & -t & 0
\end{array}\right)
$$

and

$$
O=\left(\begin{array}{cccc}
1 & 0 & F & 0 \\
0 & 1 & 0 & F \\
F & 0 & 1 & 0 \\
0 & F & 0 & 1
\end{array}\right) .
$$

The ground-state energy for the Hamiltonian is obtained by diagonalization:

$$
E_{g r}=\frac{\Delta-\left[\Delta^{2}+4\left(1-F^{2}\right)\left(t^{2}+F t(\Delta-F t)\right)\right]^{1 / 2}}{2\left(1-F^{2}\right)} .
$$

We now define the DEX interaction energy to be

$$
J_{D E X}=E_{\uparrow \downarrow}-E_{\uparrow \uparrow}
$$

where $E_{\uparrow \uparrow}\left(E_{\uparrow \downarrow}\right)$ is the ground-state energy of the system for the parallel (anti-parallel) alignment of the Mn core spins. The $E_{\uparrow \uparrow}$ is given by equation (6) while $E_{\uparrow \downarrow}$ is obtained by putting $t=0$ in the same expression, following Anderson-Hasegawa's $t \cos (\theta / 2)$ dependence of the hopping parameter $t$. Here $\theta$ is the angle between the two Mn core spins, which are treated as classical spins with fixed orientations following earlier authors.

We consider several limits.

(i) The Anderson-Hasegawa limit. In this limit, obtained by taking $\Delta \rightarrow 0$, the electron can hop between the two Mn atoms without reference to the oxygen configurations, so that the electronic motion becomes decoupled from the nuclear motion and one recovers the familiar Anderson-Hasegawa result $J_{D E X}=t$.

(ii) The Kresin-Wolf 'Diabatic'limit. In this limit, which may be obtained by putting $\Delta \rightarrow \infty$ and $F \neq 0$ in equation (6), the effect of the higher-energy configurations, $|\alpha b\rangle$ and $|\beta a\rangle$, are neglected, so that the state of the system is given by the linear combination of the two lower-lying configurations: $c_{1}|\alpha a\rangle+c_{2}|\beta b\rangle$. This is equivalent to the so-called 'diabatic' approximation and we do indeed recover the Kresin-Wolf result $J_{D E X}=F t$ in this case. [9]

(iii) The strong static JT distortion limit. Take limits $\Delta \rightarrow \infty$ and $F \sim \mathrm{e}^{-\Delta} \rightarrow 0$. We find that in this limit $J_{D E X} \rightarrow-t^{2} / \Delta$ in contrast to the linear $t$-dependence of the AndersonHasegawa DEX. Furthermore, the double-exchange process is ineffective $\left(J_{D E X} \rightarrow 0\right)$ in this limit, as the electron becomes trapped on one of the two Mn atoms, severely inhibiting the electron transfer process. The DEX interaction energies obtained from equations (6) and (7) are shown in figure 2.

It is instructive to solve for the ground-state energies using the adiabatic approximation (which we also call the Born-Oppenheimer approximation) [10]. It translates into the following steps. (i) First solve the electronic problem $(2 \times 2$ Hamiltonian and overlap matrices) for fixed nuclear configurations $\alpha$ or $\beta$. (ii) Take the energy of the lowest-energy electronic state which is the 'adiabatic potential' for the nuclear motion. (iii) Solve the nuclear problem in the adiabatic potential (also $2 \times 2$ Hamiltonian and overlap matrices). The final result is given by:

$$
E_{\text {adiabatic }}=\frac{\left(\Delta-\sqrt{\Delta^{2}+4 t^{2}}\right) / 2-F t}{1+2 A B F}
$$




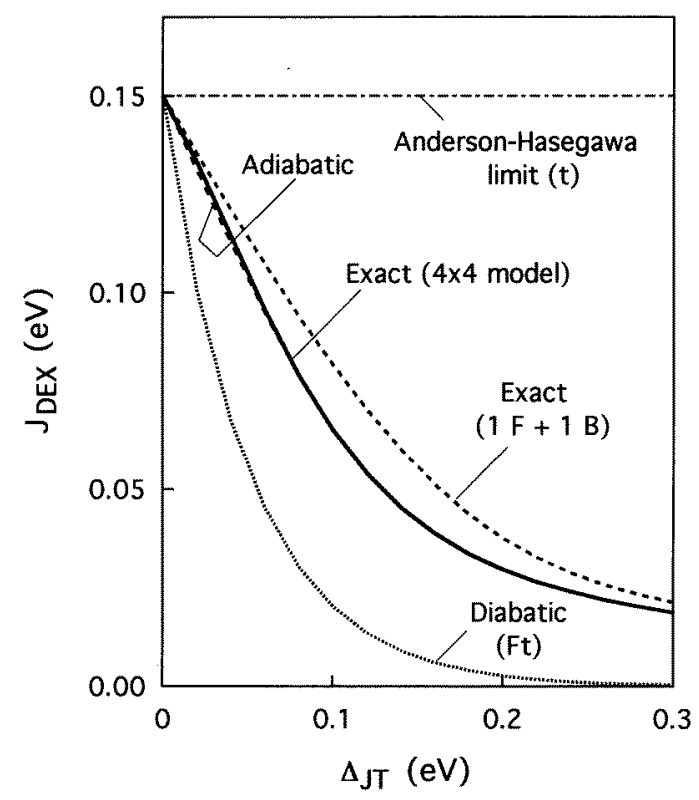

Figure 2. The double-exchange energy as a function of the JT coupling strength. Also shown are the results of the 'diabatic' approximation, the adiabatic approximation, and the 'one-Fermionone-Boson' $(1 \mathrm{~F}+1 \mathrm{~B})$ model as discussed in the text. The parameters are: $t=0.15 \mathrm{eV}$ and $\hbar \omega=0.1 \mathrm{eV}$.

where $A, B$ are the components of the normalized ground-state wavefunction of the electronic Hamiltonian

$$
H_{e}=\left(\begin{array}{cc}
0 & -t \\
-t & \Delta
\end{array}\right)
$$

The DEX interaction energy obtained by using the adiabatic energy, equation (8), instead of the exact energy, equation (6), is also shown in figure 2. The 'adiabatic' approximation is remarkably accurate in the present case, in essence because the higher 'adiabatic potential surface' is too high in energy to make a significant contribution.

In addition to the basis states $|\alpha\rangle$ and $|\beta\rangle$ corresponding to the SHO ground-state wavefunctions about each of the two nuclear configurations, if we also retain the higherenergy basis states in the Hamiltonian, the problem reduces to the so-called 'one-Fermionone-Boson' problem:

$$
H=-t\left(c_{a}^{\dagger} c_{b}+c_{b}^{\dagger} c_{a}\right)+\hbar \omega a^{\dagger} a+\xi\left(a+a^{\dagger}\right)\left(c_{a}^{\dagger} c_{a}-c_{b}^{\dagger} c_{b}\right)
$$

written in terms of the creation and annihilation operators for the SHO $\left(a^{\dagger}, a\right)$ corresponding to the potential well of the $\mathrm{O}_{6}$ octahedron and those for the electron states $\left(c_{a}^{\dagger}, c_{a}, c_{b}^{\dagger}, c_{b}\right)$. Here $\xi^{2} \equiv \hbar \omega \Delta_{J T}$. This case is much less pedagogical, but is still exactly solvable by numerical diagonalization. The detailed solution will be discussed elsewhere [11], however, the results from that model are also shown in figure 2 for the sake of comparison. We find that the energetics are not drastically altered so that the simple $4 \times 4$ Hamiltonian model presented here suffices.

We now turn to the isotope shift. In the $\mathrm{La}-\mathrm{Ca}-\mathrm{Mn}-\mathrm{O}$ systems, a reduction of $\Delta T_{c}$ of about $20 \mathrm{~K}$ is seen when ${ }^{16} \mathrm{O}$ is replaced by ${ }^{18} \mathrm{O}$. The isotope exponent $\alpha$ decreases with increasing ionic radius $\left\langle r_{A}\right\rangle$ of the cation site indicating a dependence of $\alpha$ on the electronic 
hopping parameter $t$. The only parameter in our Hamiltonian that depends on the oxygen mass is the overlap integral $F$ between the basis states $|\alpha\rangle$ and $|\beta\rangle$ describing the nuclear vibrations. The isotope substitution modifies the overlap integral thereby affecting $J_{D E X}$.

We can estimate the isotope exponent $\alpha\left(T_{c} \propto M^{-\alpha}\right)$, as observed by Zhao et al [1] from the variation of $J_{D E X}$ with the oxygen mass from the relation, $\alpha=\mathrm{d} \ln T_{c} / \mathrm{d} \ln M=$ $\mathrm{d} \ln J_{D E X} / \mathrm{d} \ln M$, where we have used the relationship $T_{c} \propto J_{D E X}$. In the diabatic limit since $J_{D E X}=F t$, the exponent is given by $\alpha=-(M / \Delta M)\left(\Delta T_{c} / T_{c}\right)=$ $-(M / \Delta M)(\Delta F / F)$, whose magnitude is too high, $\sim 1.2$ or so, for the Hamiltonian parameters we have used. As shown in figure 3, the magnitude of the exponent calculated using the exact results, equation (6), is much lower, $\alpha \sim 0.2$ for typical parameters $\left(t=0.15 \mathrm{eV}\right.$ and $\left.\Delta_{J T}=0.15 \mathrm{eV}\right)$, in reasonable agreement with the experimental results [1]. In addition, unlike the results of the diabatic approximation, the isotope exponent sharply depends on the electronic hopping parameter $t$. Physically, this dependence is expected since it is the relative magnitude of $t$ versus $\Delta_{J T}$ that determines the extent of the dynamical JT effect.

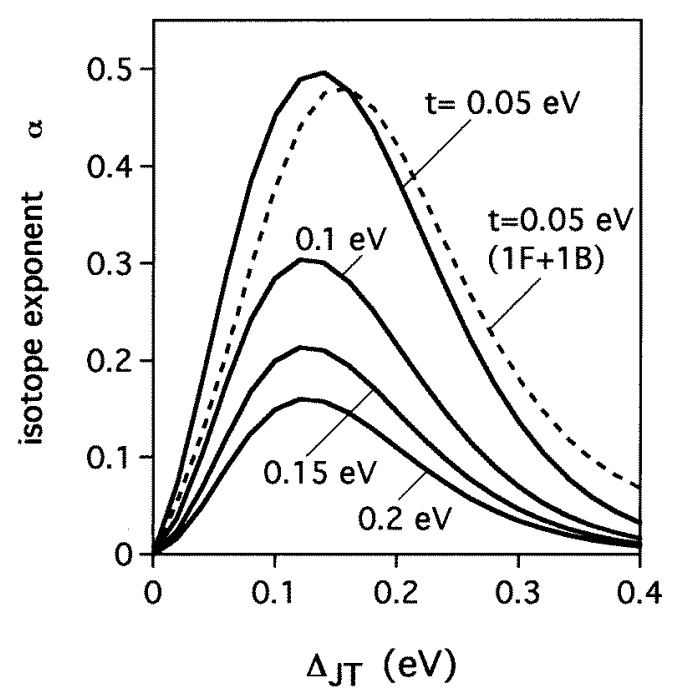

Figure 3. Dependence of the isotope exponent $\alpha$ on the Jahn-Teller coupling strength and electron hopping parameter. Solid lines represent the results for the $4 \times 4$ model Hamiltonian, while the dashed line corresponds to the result for the 'one-Fermion-one-Boson' model as discussed in the text. The zero-point energy parameter is taken to be $\hbar \omega / 2=0.05 \mathrm{eV}$ for ${ }^{16} \mathrm{O}$.

In conclusion, we have introduced a minimal $4 \times 4$ Hamiltonian model that contains the key features of the dynamical JT effect on the double-exchange interaction. The model illustrates the drastic effect of the dynamical JT coupling on the double-exchange interaction and provides a pedagogical description of the isotope effect.

I thank Peter Pfeifer for stimulating discussions and the Office of Naval Research for partial financial support under contract No ONR N00014-95-1-0439.

\section{References}

[1] Zhao G-M, Conder K, Keller H and Müller K A 1996 Nature 381676 
[2] Zhou J S and Goodenough J B 1998 Phys. Rev. Lett. 802665

[3] Millis A J 1998 Nature 392147

[4] Zener C 1951 Phys. Rev. 82403

[5] Anderson P W and Hasegawa H 1955 Phys. Rev. 100675

[6] De Gennes P-G 1960 Phys. Rev. 118141

[7] Satpathy S, Popović Z S and Vukajlović F R 1996 Phys. Rev. Lett. 76960

Pickett W E and Singh D J 1996 Phys. Rev. B 531146

[8] Elemans J B A A, van Laar B, van der Veen K R and Loopstra B O 1971 J. Solid State Chem. 3238

[9] Kresin V Z and Wolf S A 1997 Phil. Mag. B 76241

[10] Bersuker I B and Polinger V Z 1989 Vibronic Interactions in Molecules and Crystals (New York: Springer)

[11] Satpathy S, unpublished 